\title{
Transient facial paralysis after myringotomy and ventilation tube insertion under sedation with sevoflurane inhalation and four-quadrant blocks with lidocaine: a case report
}

\author{
Hyunjee Kim', Joonhee Lee', Younghoon Jeon ${ }^{1,2}$ \\ 1Department of Anesthesiology and Pain Medicine, Kyungpook National University Hospital, Daegu, Korea \\ ${ }^{2}$ Department of Anesthesiology and Pain Medicine, School of Dentistry, Kyungpook National University, Daegu, Korea
}

\begin{abstract}
Myringotomy and ventilation tube insertion are widely performed in pediatric patients with chronic otitis media. This procedure is performed under general anesthesia or sedation with local anesthesia infiltration in pediatric patients. In this case report, we report a case of transient facial paralysis in a pediatric patient who underwent myringotomy and ventilation tube insertion using sevoflurane inhalation and four-quadrant blocks with lidocaine.
\end{abstract}

Keywords: Facial Paralysis; Local Anesthetics; Myringotomy; Otitis Media.

This is an Open Access article distributed under the terms of the Creative Commons Attribution Non-Commercial License (http://creativecommons.org/licenses/by-nc/4.0/) which permits unrestricted non-commercial use, distribution, and reproduction in any medium, provided the original work is properly cited.

\section{INTRODUCTION}

Myringotomy and insertion of a tympanostomy tube are performed to release middle ear fluid in pediatric patients with chronic otitis media (COM). Drainage of effusion in the middle ear via a small incision in the tympanic membrane leads to resolution of COM. This procedure is performed under general anesthesia or sedation combined with infiltration of local anesthetic $[1,2]$. In this case report, we report a pediatric patient with transient facial paralysis after myringotomy and insertion of a ventilation tube using sevoflurane inhalation and local infiltration with lidocaine.

\section{CASE REPORT}

A 2-year-old boy was admitted for myringotomy and ventilation tube insertion. The patient had a one-year history of left ear COM. The patient's height was 103 $\mathrm{cm}$ and body weight was $15 \mathrm{~kg}$. He had no unremarkable medical history besides COM, which caused moderate hearing loss (50-60 dB). In addition, his preoperative laboratory test and chest X-ray showed no abnormalities.

A 23-gauge cannula was placed on the radial side of the right wrist after topical anesthesia with a eutectic mixture of lidocaine $2.5 \%$ and prilocaine $2.5 \%$ (EMLA). Monitoring (electrocardiography, pulse oximetry, noninvasive blood pressure monitoring, and capnography) was implemented. The patient received an intravenous

Received: April 14, 2020 • Revised: May 10, 2020 • Accepted: June 1, 2020

Corresponding Author: Younghoon Jeon, Department of Anesthesiology and Pain Medicine, School of Dentistry, Kyungpook National University, 130 Dongdeok-ro, Jung-gu, Daegu 41944, Korea

Tel: +82-53-200-5871 Fax: +82-53-426-2760 E-mail: jeon68@gmail.com

Copyright@ 2020 Journal of Dental Anesthesia and Pain Medicine 
injection of ketamine $15 \mathrm{mg}$. Manual assisted ventilation using a face mask was initiated with sevoflurane 2-3 vol $\%$ and $60 \%$ oxygen, and spontaneous ventilation was maintained during the operation. Lidocaine $1 \%$ with epinephrine (1: 10,000) was infiltrated through the skin overlying the cartilaginous meatus of the left ear. The myringotomy and ventilation tube insertion in the left ear were performed. The electrocardiography had a normal sinus rhythm, systolic blood pressure was between 95$100 \mathrm{mmHg}$, diastolic pressure was $52-56 \mathrm{mmHg}$, heart rate was $135-139$ beats/min, pulse oximetry was $100 \%$, and end-tidal $\mathrm{CO} 2$ was 35 , respectively, during the operation. The duration of the operation was $20 \mathrm{~min}$. After the completion of myringotomy and insertion of a tympanostomy tube, $100 \%$ oxygen was provided to facilitate consciousness recovery. There were no complications during the emergence, and time until eye-opening was $6 \mathrm{~min}$. The patient was transferred to a post-anesthetic care unit, and a drooping of the left corner of the patient's mouth was found (Fig. 1). However, the facial paralysis completely subsided within $2 \mathrm{~h}$ after surgery. Consent for publication of this report was obtained from the patient's families.

\section{DISCUSSION}

Myringotomy with tube placement is one of the most frequently performed surgeries in pediatric patients with COM. It is a very painful procedure requiring dense anesthesia. Therefore, this procedure is performed under general anesthesia or sedation with local anesthesia infiltration in pediatric patients $[1,2]$.

The facial nerve stems from the pons and travels through the internal acoustic meatus of the petrosal bone and courses through the facial canal. The facial nerve continues to travel through the stylomastoid foramen and enters the parotid gland, where it divides into four branches. The temporal and zygomatic branches pass upward or behind the ramus of the mandible and buccal, and the mandibular branch travels downwards behind



Fig. 1. Drooping left corner of patient's mouth following four quardant block with lidocaine

mandibular ramus.

Transient facial palsy associated with mask anesthesia is very rare but can occur $[3,4]$. Digital pressure behind the mandible or excessive pressure exerted by the facemask to facilitate ventilation can cause a traumatic injury of the facial nerve. Generally, resolution of facial palsy following mask anesthesia takes several weeks $[3,4]$.

Four-quadrant blocks with local anesthetics are frequently used for pain control during myringotomy combined with tube insertion [5]. The facial nerve travels through the internal acoustic meatus of the petrosal bone, which is closely related to the middle ear cavity. The middle ear cavity is the portion between the eardrum and the oval window of the internal acoustic cavity. Four-quadrant block anesthesia of the external auditory canal is performed by subcutaneous injection of local anesthetics into the four quadrants of the lateral portion of the canal. Therefore, local anesthetics following a four-quadrant block can be diffused into the middle ear, which may lead to acute ipsilateral facial paralysis [6]. Hoffman and Li reported two cases of transient facial palsy after a four-quadrant block [6]. However, the overall incidence of facial nerve palsy following four-quadrant block remains unknown. The complete 
resolution of facial nerve palsy by infiltration of local anesthetics takes a few hours [6]. Facial palsy can occur after a viral infection, such as herpes zoster and influenza $\mathrm{B}$, and trauma. However, the patient showed no rash and upper respiratory infection symptoms. In addition, there were no bruises or scars in the head and face region. In this case, the patient showed drooping of the left corner of mouth. The onset was sudden and painless. The corneal light reflex was normal, and no neurologic abnormalities were found.

In this case, the facial palsy completely resolved within $2 \mathrm{~h}$ after surgery. Therefore, we presumed that the four-quadrant block with lidocaine caused the transient facial palsy.

In conclusion, facial nerve palsy associated with infiltration of local anesthetic in the external ear meatus is very rare but has an excellent prognosis. However, the patient and the patient's family can become very anxious and irritable until the symptoms resolve. Therefore, anesthesiologists should be aware of this possibility to reassure patients and provide information on facial nerve palsy associated with four-quadrant blocks with local anesthetics.

\section{AUHOR ORCIDS}

Younghoon Jeon: https://orcid.org/0000-0002-7168-4214

Hyunjee Kim: https://orcid.org/0000-0002-6336-7622

Joonhee Lee: https://orcid.org/0000-0001-6566-4287

\section{AUHOR BONHBIBIIONS}

Younghoon Jeon: Conceptualization and editing

Hyunjee Kim: Writing

Joonhee Lee: Data curation

CONFLICT OF INTEREST: No conflicts of interests and no funding.

\section{REFERENCES}

1. Storrs LA. Topical anesthesia for myringotomy. Laryngoscope 1968; 78: 834-9.

2. Robinson H, Engelhardt T. Ambulatory anesthetic care in children undergoing myringotomy and tube placement: current perspectives. Local Reg Anesth 2017; 10: 41-9.

3. Ananthanarayan C, Rolbin SH, Hew E. Facial nerve paralysis following mask anaesthesia. Can J Anaesth 1988; 35: $102-3$.

4. Glauber DT. Facial paralysis after general anesthesia. Anesthesiology 1986; 65: 516-7.

5. Hirsch C. 50 years of local anesthesia in otolaryngology. Acta Otolaryngol 1934; 21: 256-78.

6. Hoffman RA and Li CL. Tetracaine topical anesthesia for myringotomy. Laryngoscope 2001; 111: 1636-8. 